\title{
Temperature dependence of Gilbert damping in manganite/normal metal heterostructure
}

\author{
Timur A. Shcaihulov ${ }^{*}$, Victor V. Demidov, Igor V. Borisenko, Gennady A. Ovsyannikov \\ Kotel'nikov IRE RAS, Mokhovaya 11-7, Moscow 125009, Russia
}

\begin{abstract}
The temperature dependence of the spin-pumping effect on the Gilbert damping in a bilayer based on epitaxial manganite film grown on neodymium galate substrate was investigated by measuring of the linewidth of the ferromagnetic resonance spectrum (FMR). Ferromagnetic resonance in thin ferromagnetic manganite $\mathrm{La}_{0.7} \mathrm{Sr}_{0.3} \mathrm{MnO}_{3}$ (LSMO) films is used to produce a spin current at the interface between a metallic and a ferromagnetic layer. Pt/LSMO/NGO, Au/LSMO/NGO and LSMO/NGO heterostrucutres were measured. An increase of the linewidth in FMR spectrum at the temperature lower liquid nitrogen temperature $(77 \mathrm{~K})$ is observed
\end{abstract}

\section{Introduction}

The operation of the most promising spintronics devices is based on a spin transport in magnetic heterostructures associated with the transfer of the spin moment when a spin-polarized current flows through them. Magnetic heterostructure, as a rule, consists of magnetic and nonmagnetic layers. Rare-earth manganite perovskites with the structure $\operatorname{Re}_{1-\mathrm{x}} \mathrm{A}_{\mathrm{x}} \mathrm{MnO}_{3}$ (Re is-rare-earth materials of the type $\mathrm{La}$ or $\mathrm{Nd}$ ), and $\mathrm{A}$ is alkaline earth metals such as $\mathrm{Sr}, \mathrm{Ca}, \mathrm{Ba}$ ) exhibit a wide range of unusual electrical and magnetic properties, including the colossal magnetoresistance effect (see, review [1]). The parameters of epitaxial films of these materials very often differ substantially from the properties of single crystals. A significant influence on the magnetic and electrical properties of the films is caused by the strain in the films caused by a mismatch with the substrate on which the manganite film [1-3] is deposited, as well as the effects of phase separation and the presence of a nonmagnetic layer at the substrate/film interface [4], which are the strongest Appear in very thin films (less than $10 \mathrm{~nm}$ ). The lanthanum-strontium manganites $\mathrm{La}_{0.7} \mathrm{Sr}_{0.3} \mathrm{MnO}_{3}$ (LSMO) have high spin polarization (up to $100 \%$ ) and can be used in magnetic tunnel junctions $[5,6]$ and spin valves $[7,8]$. Manganite films for which the Curie temperature Tc is close to room temperature, especially attractive for practical applications. Although a number of studies have been carried out on the excitation of a spin current by ferromagnetic resonance in two LSMO/NM layer structures (NM is a normal metal, usually platinum) $[9,10]$, there is no data on the temperature dependence of the width of the FMR line in the presence of spin current in ferromagnets.

\section{Model for spin current}

Due to phonon or magnon scattering an infinite precession of the magnetization around the external magnetic field reaches a stable equilibrium state parallel to the effective magnetic field. For a correct description of this phenomena Gilbert proposed introduced a phenomenological correction responsible for the dissipation in the magnetic dynamics [11], which leads to the Landau-Lifshitz-Gilbert equation (LLG):

$$
\frac{d \vec{m}}{d t}=-\gamma_{0} \vec{m} \times \vec{H}_{e f f}+\alpha \vec{m} \times \frac{d \vec{m}}{d t}
$$

here $\alpha$ is the Hilbert damping coefficient, $\mathrm{m}=\mathrm{M} /|\mathrm{M}|$ is unit magnetization vector of ferromagnetic layer, $\gamma=\mathrm{g} \mu_{\mathrm{B}} / \mathrm{h}$ is the gyromagnetic ration, $\mathrm{H}_{\text {eff }}$ is $\mathrm{s}$ the local effective magnetic field including the external, demagnetization and crystal anisotropy field. The Gilbert damping $\alpha$ is the times scale for relaxation which can take place in two different ways: either the spin angular momentum is transferred to non-magnetic degrees of freedom, which is called direct, homogenous damping; or the spin angular momentum is transferred to other magnetic degrees of freedom such as spin waves, impurity, which is called indirect Inhomogeneous dampingm Significant relaxation processes in thin ferromagnetic films [12] are spin-orbit relaxation damping, two-magnon scattering and eddy current damping.

The increase in the Gilbert damping parameter in a heterostructure from a ferromagnetic and a normal (nonmagnetic) metal caused by precession of the magnetization in a ferromagnetic that causes the spin current to flow through the boundary into a normal metal was considered theoretically [13]. The theory explains the injection of a spin current from a ferromagnetic to a nonmagnetic metal by scattering of the spin at the 
interface. The spin current from ferromagnetic to the non-magnetic layer moves perpendicular to the interface

$$
j_{s}^{0} \vec{s}=\frac{\hbar}{8 \pi} \operatorname{Re}(2 g \uparrow \downarrow)\left[\vec{m} \times \frac{\partial m}{\partial t}\right]
$$

where $\operatorname{Re}(2 \mathrm{~g} \uparrow \downarrow)$ is the spin mixing conductance which is additively added to the components of the Gilbert damping.

The Gilbert damping parameter can be written as $\alpha=\alpha_{0}+\alpha^{\prime}$, where $\alpha_{0}$ is the intrinsic contribution and $\alpha^{\prime}$ is the additional damping due to spin pumping $[13,14]$

$$
\alpha^{\prime}=\frac{\not \hbar g^{\uparrow \downarrow}}{4 \pi M_{0} t_{F M}}
$$

\section{Samples and experimental technique}

Epitaxial films $\mathrm{La}_{0.7} \mathrm{Sr}_{0.3} \mathrm{MnO}_{3}$ (LIMO) were deposited by magnetron sputtering on the (110) plane of a singlecrystal substrate of $\mathrm{NdGaO} 3$ (NGO). 10-20 nm of Pt or $\mathrm{Au}$ were sputtered ex situ just after cooling of the film. Contact areas were produced by sputtering of Pt through a metal mask. The resistance of the films was studied by a four-point method, which excludes the influence of contact resistance.

The magnetic characteristics were measured by magnetic resonance technique using ER-200 EPR spectrometer from Bruker (frequency $9.76 \mathrm{GHz}$ ). When recording the signals, a synchronous detection was used at the modulation frequency of an external magnetic field, the value of which was $100 \mathrm{kHz}$. The investigated samples were located in the microwave cavity of the spectrometer in such a way that the plane of the sample was always parallel to the direction of the constant external magnetic field and the magnetic component of the microwave field (parallel orientation). The parallelism of the plane of the samples to a constant magnetic field was controlled by the minimum of the resonance value of the field of the ferromagnetic resonance (FMR) lines). This arrangement of the samples eliminated the change in the magnetic resonance spectra due to shape anisotropy. Rotation of the samples was carried out around an axis perpendicular to the plane of the samples.

The films just after deposition ( is designd as dLSMO) and heated at $\mathrm{T}=720 \mathrm{C}$ during one hour (is designed as h-LSMO) were investigated

\section{Magnetic parameters of the films}

The method for determining the parameters of the magnetic anisotropy consists in processing the angular dependences of the resonant fields of the FMR spectra. The solution of the Landau-Lifshitz equation for the evolution of the magnetization $\mathrm{M}$ in an external constant magnetic field $\mathrm{H}$ under the action of the magnetic component of the radio-frequency field is used, which gives an analytic equation for the resonance field $\mathrm{H}_{0}$ and the frequency $\omega[15]$

$$
\begin{aligned}
& \left(\frac{\omega}{\gamma}\right)^{2}=\left(4 \pi M_{0}+H_{0}+\frac{2 K_{u}}{M_{0}} \cos ^{2} \varphi_{u}+\frac{2 K_{c}}{M_{0}} \frac{1+\cos ^{2} 2 \varphi_{c}}{2}\right) \\
& \left(H_{0}+\frac{2 K_{u}}{M_{0}} \cos 2 \varphi_{u}+\frac{2 K_{c}}{M_{0}} \cos 4 \varphi_{c}\right)
\end{aligned}
$$

here $\gamma$ is the gyromagnetic ratio, $\mathrm{M}_{0}$ is equilibrium magnetization, $\mathrm{K}_{\mathrm{u}}$ and $\mathrm{K}_{\mathrm{c}}$ are the uniaxial strain and the biaxial cubic anisotropy constants correspondingly. $t \varphi_{\mathrm{u}}$ and $\varphi_{\mathrm{c}}$ are the angles between the earsy axis of the uniaxial and cubic anisotropies and the external magnetic field correspondingly. $\mathrm{K}_{\mathrm{u}}, \mathrm{K}_{\mathrm{c}}, \mathrm{M}_{0}$ as well as angles $\varphi_{\mathrm{u}}$ and $\varphi_{\mathrm{c}}$ were obtained by the fitting of the angular dependence (Fig.1)

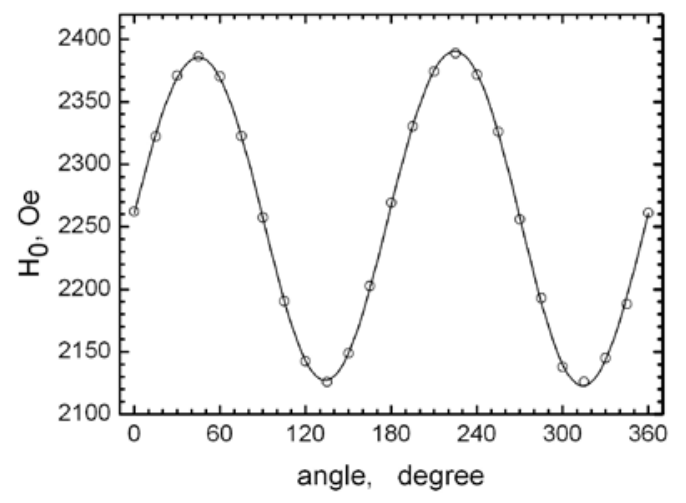

Fig. 1. Angular dependence of resonance field for hLSMO film at $\mathrm{T}=300 \mathrm{~K}$. The fitting of the experimental data using eq. (3) give the following magnetic parameters of LSMO film: magnetization $\mathrm{M}_{0}=300 \mathrm{Oe}$, $\mathrm{H}_{\mathrm{u}}=190 \mathrm{Oe}, \mathrm{H}_{\mathrm{c}}=10 \mathrm{Oe}$

Fig. 1 shows the angular dependence of the resonance value of the magnetic field $\mathrm{H}_{0}$ for the LSMO film taken at room temperature and which was rotated around the normal to the film plane.pthe sample by an angle $\varphi$. The angle was measured from one of the faces of the substrate. The constant magnetic field and the magnetic component of the microwave field were in the plane of the film. Since the substrate with the film was square $5 \times 5 \mathrm{~mm}$, the effect of the anisotropy of the shape of the sample is minimal, and the entire shift of the resonant field is due to the planar magnetic anisotropy of the LBMO film. The angular dependence was described by a resonance relation taking into account magnetic uniaxial and cubic plane anisotropies (3) [15]. It can be seen that the relationship used describes the experimental data quite well. As a result, the magnetization $\mathrm{M}_{0}$, anisotropy constants $\mathrm{Ku}$ and $\mathrm{Kc} \mathrm{H}_{\mathrm{u}, \mathrm{c}}$ $=2 \mathrm{~K}_{\mathrm{U}, \mathrm{C}} / \mathrm{M}_{0}$ were determined.

The temperature dependences of magnetic parameter are shown on Fig.2. Magnetization of the film increases up to $2.2 \mu_{\mathrm{B}} / \mathrm{Mn}$ at $\mathrm{T} \leq 200 \mathrm{~K}$. Magnetic anisotropy fields increase at lower $\mathrm{T}<50 \mathrm{~K}$. Uniaxial magnetic anisotropy dominates over cubic anisotropy. 


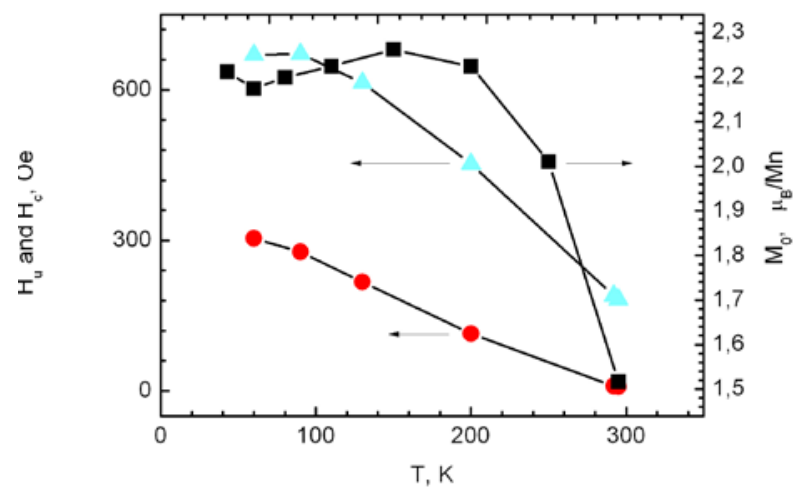

Fig.2. Temperature dependence of magnetization $\mathrm{M}_{0}$, biaxial $\mathrm{H}_{\mathrm{u}}$ and cubic $\mathrm{H}_{\mathrm{c}}$ magnetic field anisotropy correspondingly for h-LSMO film

\section{The linewidth of FMR}

The width of the ferromagnetic resonance line (FMR), measured during the scanning of an external magnetic field $\mathrm{H}$, is defined as the difference betweem the extrema of the spectrum.

$$
\delta_{\mathrm{pp}}=\mathrm{H}_{\mathrm{p}}+-\mathrm{H}_{\mathrm{p}-}
$$

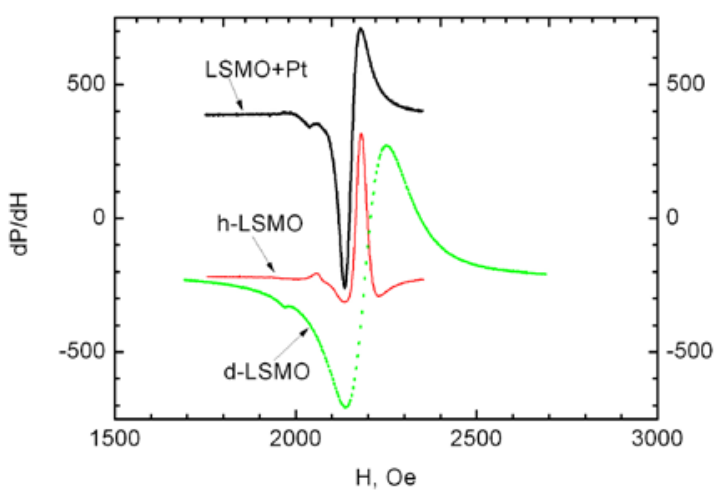

Fig. 3. FMR spectrum for d-LSMO and Pt/LSMO heterostructures. The increasing of linewidth is clearly observed. The spectra for d-LSMO and Pr/h-LSMO are moved on the axis $\mathrm{dP} / \mathrm{dH}$.

The initial and final points of which $\mathrm{H}_{\mathrm{p}+}$ and $\mathrm{H}_{\mathrm{p}-}$ constitute the position of the maximum and minimum of the first derivative $\mathrm{dP} / \mathrm{dH}$ of the high-frequency field absorption signal $\mathrm{P}(\mathrm{H})$ (see Fig. 2). At this value of the resonance field, $\mathrm{H}_{0}$, defined as the transition point of the signal $\mathrm{dP} / \mathrm{dH}$ through zero, always lies in the range of $\mathrm{H}_{\mathrm{p}}$ $+<\mathrm{H}_{0}<\mathrm{H}_{\mathrm{p}}$. It is known that the linewidth $\delta_{\mathrm{pp}}$ determined in the above way depends on the angle $\varphi$ that determines the orientation of the resonant magnetic field with respect to axes of anisotropy.

The physical reason for the change in linewidth $\Delta \mathrm{H}_{\mathrm{pp}}$ films of anisotropic ferromagnets due to the change of the equilibrium orientation of the magnetization $\mathrm{M}$ with the registration of the absorption line. It is known that in manganite films contribution to the angular dependence of $\mathrm{H}_{0}$ are making as uniaxial (substrate influence) and cubic (crystallographic) magnetic anisotropy. When the deviation of the magnetic field from the axis of the anisotropy of the linewidth of the FMR increases .

We deposited normal metal $(\mathrm{Pt}, \mathrm{Au})$ on LSMO to evaluate the efficiency of generation of spin current at the boundary of a ferromagnetic-normal metal. Using the following expressions, we calculated the spin mixing conductance in bilayers LSMO/Pt.

$$
g_{\text {eff }}^{\uparrow \downarrow}=\frac{4 \pi \gamma_{g} M_{s} t_{L S M O}}{g \mu_{\beta} \omega_{f}}\left(\delta H_{P t / L S M O}-\delta H_{L S M O}\right)
$$

$\gamma_{\mathrm{g}}=17,605 \cdot 10^{6}$ is gyromagnetic ratio for electron , $\omega_{\mathrm{f}}=2 \pi \cdot 9.51 \cdot 10^{9} \mathrm{~s}^{-1}$ is the microwave angular frequency, $\mathrm{M}_{\mathrm{s}}=300 \mathrm{Oe}$ is the magnetization LSMO film, $\mathrm{t}_{\mathrm{LSMO}}=40$ $\mathrm{nm}$ is the thickness of the layer of platinum, $\mu_{\mathrm{B}}=9.274 \cdot 10^{-21} \mathrm{erg} / \mathrm{Gs}$ is the Bohr magneton, $\mathrm{g}=2$ is Lande factor. We got in the case $\delta_{\mathrm{pp}}=130 \mathrm{Oe}$ at $\mathrm{T}=60 \mathrm{~K}$ $\mathrm{g}_{\text {eff }}=1,510^{20} \mathrm{~m}^{-2}$. For comparison for interface Py/Pt it was obtained $\mathrm{g}_{\text {eff }}=2,1 \cdot 10^{19} \mathrm{M}^{-2}$ [16] and for YIG/Pt $\mathrm{g}_{\text {eff }}=4,8 \cdot 10^{20} \mathrm{M}^{-2}[17]$. There is no spin conductance in bulk Pt taken into consideration. So the obtained value of spin-conductance is over estimated.

\section{Temperature dependence}

Temperature dependence of FMR spectrum and, in particular, the width of the resonance lines of magnetization, and other parameters in the heterostructure of Pt/LSMO and LSMO were measured. Possible sources of additional damping could be the sputtered film of normal metal ( $\mathrm{Pt}$ and $\mathrm{Au}$ ) on LSMO. The mechanism of spin pumping weakens the precession of the magnetization, creating a spin current from the ferromagnetic film to the layer of normal metal[16-18]. Several other processes contribute to the temperature dependence of damping [14].

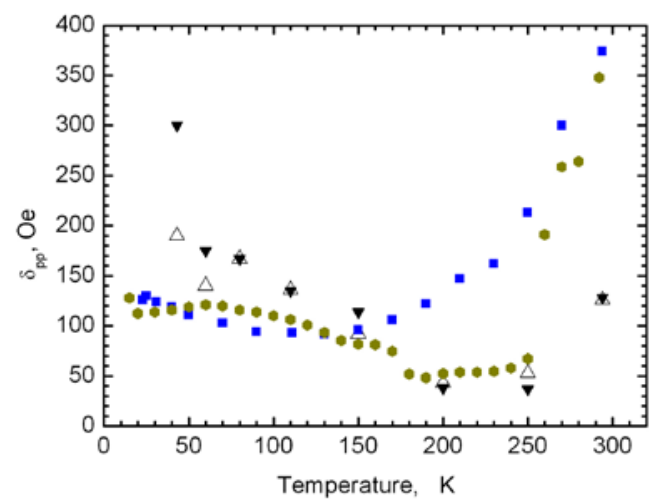

Fig.4. Temperature dependence of FMR linewidth for the following heterostuctures $\mathrm{Au} / \mathrm{d}-\mathrm{LSMO}, \mathrm{Pt} / \mathrm{d}-\mathrm{LSMO}$ (triangle), d-LSMO (empty triangle, inverse triangle) for two angles of magnetic field directions 
The temperature dependence of the FMR linewidth for the heterostructures based on d-LSMO are given in figure 4 . It is seen that with decreasing temperature the linewidth of all the structures is reduced. The increase in linewidth in the heterostructure $\mathrm{Au} / \mathrm{LSMO} / \mathrm{NGO}$ at low temperatures $\mathrm{T}<150 \mathrm{~K}$ can be attributed to existence the layer with the reduction of Curie temperature at the interface LSMO with substrate. It plays the role of a barrier which blocks the spin pumping interface at $\mathrm{T}$ low TCU of interfaced. The effect was observed for heterostructure $\mathrm{Pt} / \mathrm{SRO} / \mathrm{LSMO}$. Here $\mathrm{SRO}$ is $\mathrm{SrRuO} 3$ film with $\mathrm{T}_{\mathrm{CU}}=150 \mathrm{~K}[19]$.

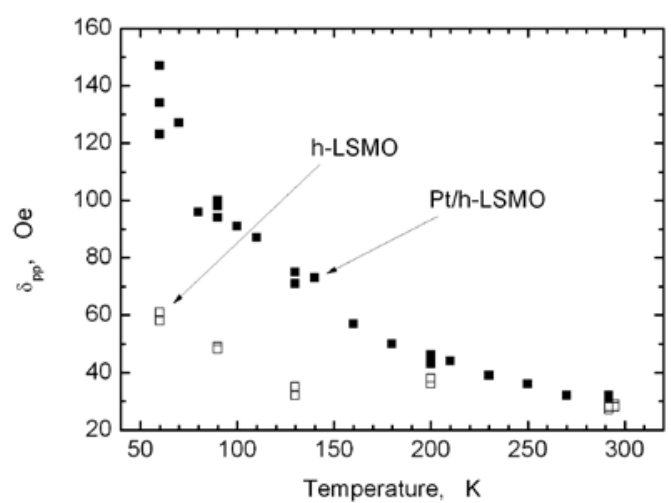

Fig.5. Temperature dependence of FMR linewidth for the films h-LSMO, and Pt/h-LSMO taken with an external magnetic field directed along the easy axis of uniaxial magnetic anisotropy

The temperature dependences of linewidth for the manganite film h-LSMO and for Pt covered Pt/h-LSMO are presented at Fig. 5. There is no reduction for linewidth at high $\mathrm{T}$ observed. Comparison data on Fig.4 and Fig.5 show that inhomogeneous increasing of line width clearly observed at high temperature $\mathrm{T}>150 \mathrm{~K}$. Increasing of linewidth at low $\mathrm{T}$ still exist even at hLSMO which more homogeneous then d-LSMO. Here possible mechanism increasing of linewidth with $\mathrm{T}$ is connected with interface at the substrate and LSMO film.

\section{Conclusion}

The temperature dependence of FMR linewidth and magnetic parameters of LSMO and Pt/LSMO films were investigated. From linewidth obtained for the film before and after deposition of Pt film on top of epitaxial LSMO films the spin mixing conductance was determined. The non monotonic dependence of linewidth of FMR spectrum was observed; decreasing of linewidth at high temperature and increasing at $\mathrm{T}<150 \mathrm{~K}$. The increase in linewidth in the heterostructure $\mathrm{Au} / \mathrm{LSMO} / \mathrm{NGO}$ at low temperatures $\mathrm{T}<150 \mathrm{~K}$ can be attributed to existence the layer with the reduction of Curie temperature at the interfaces of LSMO either with normal metal or substrate.
V.A. Atsarkin is acknowledged for fruitful discussion. Support by RFBR 17-02-00145, 16-29-14022 and Scientific school NSh -8168.2016.2.

\section{References}

1. A.-M. Haghiri-Cosnet and J.P. Renard, J. Phys D:Appl. Phys. 36, R127 (2003).

2. Zh. Huang, G.Y. Gao, Zh.Zh. Yin, X.X. Feng, Y.Zh. Chen, X.R. Zhao, J.R. Sun, W.B. Wu, J. Appl. Phys. 105, 113919 (2009).

3. G.A. Ovsyannikov, A.M. Petrzhik, I.V. Borisenko, A.A. Klimov, Yu.A. Ignatov, V.V. Demidov, S.A. Nikitov, JETP, 108, 48 (2009).

4. N.D. Mathur, G. Burnell, S.P. Isaac, T.J. Jackson, B.S. Teo, J.L. MacManus-Driscoll, L.F. Cohen, J.E. Evetts, M.G. Blamire, Nature 387, 266 (1997).

5. M. Bowen, M. Bibes, A. Barthélémy, J.P. Contour, A. Anane, Y. Lemaître, and A. Fert, Appl. Phys. Lett. 82, 233 (2003).

6. Y. Ishii, H. Yamada, H. Sato, H. Akoh, M. Kawasaki, and Y. Tokura, Appl. Phys. Lett. 87, 22509 (2005).

7. Z. H. Xiong, D. Wu, Z. V. Vardeny, and J. Shi, Nature 427, 821 (2004).

8. V. Dediu, M. Murgia, F. C. Matacotta, C. Taliani, and S. Barbanera, Solid State Commun 122, 181 (2002). 9. G. Y. Luo, C. R. Chang, and J. G. Lin, J . Appl. Phys 115-119, 17C508 (2014).

10. V.A. Atsarkin, B.V. Sorokin, I.V. Borisenko, V.V. Demidov and G.A. Ovsyannikov, J. Phys. D: Appl. Phys. 49 (2016) 125003 (8pp).

11. T.L. Gilbert, IEEETrans.Magn. 40, 3443 (1949)

12. B Heinrich, R Urban and $\mathrm{G}$ Woltersdorf, J. Appl. Phys. 91, 7523 (2002)

13. A. Tserkovnyak, Brataas, E.W. Bauer, Phys. Rev. Lett., 88, 117601 (2002).

14. A. Azevedo,L.H. Vilela-Leao,R. L. RodriguezSuarez, A.F. Lacerda Santos, and S. M. Rezende Phys.Rev. B83, 144402 (2011).

15. V.V. Demidov, G.A. Ovsyannikov, A.M. Petrzhik, I.V. Borisenko, A.V. Shadrin, and R. Gunnarsson J. Appl. Phys. 113, 163909 (2013).

16. O. Mosendz, V. Vlaminck, J. E. Pearson, F.Y. Fradin, G. E. W. Bauer, S. D. Bader, and A. Hoffmann, Phys. Rev. 82, 214403 (2010).

17. M. Rezende, R. L. Rodr_1guez-Suarez, M. M. Soares, 1 L. H. Vilela-Le, D. Ley Dom_inguez, and A. Azeved, Appl.Phys.Lett. 102, 012402 (2013).

18. T.G.A. Verhagen, H.N. Tinkey, H.C. Overweg, M. van Son, M. Huber, J.M. van Ruitenbeek and J. Aarts, J. Phys. Condens. Matter 28, 056004 (2016) (10pp). 19. S. Emori, U.S. Alaan, M.T. Gray, V.Sluka, Y. Chen, A.D.Kent, Y.Suzuki, Phys. Rev B94, 224423 (2016). 